\title{
The influence on biotissue laser resection of a strongly absorbing layer at the optical fiber tip
}

\author{
Daria Kuznetsova, ${ }^{*, *}$, Vadim Elagin* and Maria Karabut ${ }^{*, \dagger}$ \\ *Institute of Biomedical Technologies \\ Nizhny Novgorod State Medical Academy \\ 10/1 Minin and Pozharsky Sq., Nizhny Novgorod 603005, Russia \\ ${ }^{\dagger}$ Lobachevsky State University of Nizhni Novgorod \\ 23 Gagarin Avenue BLDG 2, Nizhny Novgorod 603950, Russia \\ ¥daria.s.kuznetsova@gmail.com \\ Maria Shakhova \\ Nizhny Novgorod State Medical Academy \\ 10/1 Minin and Pozharsky Sq., Nizhny Novgorod 603005, Russia \\ Vladimir Bredikhin \\ Institute of Applied Physics, Russian Academy of Sciences \\ 46 Ul'yanov Street, Nizhny Novgorod 603950, Russia \\ Liudmila Snopova and Andrei Shakhov \\ Nizhny Novgorod State Medical Academy \\ 10/1 Minin and Pozharsky Sq., Nizhny Novgorod 603005, Russia \\ Natalia Sapogova and Nikita Bityurin \\ Institute of Applied Physics, Russian Academy of Sciences \\ 46 Ul'yanov Street, Nizhny Novgorod 603950, Russia \\ Victor Bagratashvili \\ Institute on Laser and Information \\ Technologies Russian Academy of Sciences \\ 2 Pionerskaya St., Troitsk 142092, Russia \\ Vladislav Kamensky \\ Institute of Applied Physics, Russian Academy of Sciences \\ 46 Ul'yanov Street, Nizhny Novgorod 603950, Russia
}

Received 3 August 2015

Accepted 9 October 2015

Published 26 November 2015

\$Corresponding author.

This is an Open Access article published by World Scientific Publishing Company. It is distributed under the terms of the Creative Commons Attribution 4.0 (CC-BY) License. Further distribution of this work is permitted, provided the original work is properly cited. 
In this paper, we consider a method of laser resection using the silica glass core from which the cladding layer has been removed as the cutting part of a laser scalpel. An absorbing layer coating the silica fiber tip markedly alters its biotissue cutting characteristics. The results of histological studies of skin after exposure to a laser scalpel with and without a strongly absorbing coating (SAC) at a wavelength of $0.97 \mu \mathrm{m}$ show that resection using a coated scalpel is more sparing. When an uncoated scalpel was used, skin injury was more apparent in both its surface spread and the depth of structural damage, resulting in poorer tissue regeneration.

Keywords: Laser surgery; silica fibers; strongly absorbing coating; histology.

\section{Introduction}

We present an experimental study aimed at developing a method of laser surgery that may be termed 'indirect' because the radiation is not immediately incident on the biotissue but on a converting element in contact with the biotissue. The tip of the laser's silica fiber glass core from which the cladding layer has been removed is used as the cutting part of the laser scalpel. This optical fiber is mechanically flexible, but its silica core is very strong and the tip is resistant to the high temperatures arising due to the carbonization of the biotissue exposed to the laser scalpel. In the tissues adjacent to the dissection, a coagulation area is formed which ensures a bloodless incision.

The optimal area of thermal damage caused within different types of tissue when using various resection speeds have been issues of primary importance in fiber laser surgery over the last decade $^{1-4}$ To optimize the conditions of exposure, various special fiber tips have been used. Some of them increase the power density due to radiation focusing, ${ }^{5}$ others, e.g., sapphire tips, ${ }^{6}$ have better thermal diffusivity. There have also been reports ${ }^{7}$ on a device for intratissue irradiation, in which tips with mirrored metal elements at the distal end deflect the light beam at the fiber output into a cylindrical pattern in order to protect healthy tissues. Also interesting are tips generating high-frequency acoustic radiation that disinfects the biotissue. ${ }^{8}$

In the present paper, we describe the use of strongly absorbing coatings (SACs), which can be easily formed in the region of contact with biotissue, and which change the temperature conditions caused by exposure to the laser scalpel. A new method of increasing biotissue absorption, using a laser fiber scalpel, has recently been developed and put into practice. ${ }^{9}$ The method is basically as follows: A destruction process with the formation of absorbing agents (carbon) is initiated during biotissue surgery but one that is later self-sustaining due to the absorption of light by the agents which have already formed. The drawback of this method is the instability of the self-sustaining process of destruction and ablation, and the need to find ways automatically to maintain the temperature in the treated area. The goal of our work was to create stable conditions of exposure to laser radiation at the fiber tip by coating the output end of the fiber used as the cutting surface so that it would retain its absorbing and mechanical properties for a long time, and also to carry out a comparative study of the features of laser resection with and without such a coating.

We investigated the method of laser resection with a silica fiber glass core cleared of its cladding layer for use as the scalpel. The biotissue is heated by the laser radiation and moved apart by the silica core of the optical fiber. ${ }^{10}$ The silica core is sufficiently transparent in the near IR region and is mechanically strong enough to stand the high temperatures arising at the tip of the optical fiber tip during biotissue resection. The strongly absorbing layer at the tip of the fiber (according to the work of Amzayyb ${ }^{11}$ the absorption of the layer can achieve 50\%) results in heating of the silica core to temperatures higher than carbonization temperature and facilitates carbon formation during biotissue dissociation resulting from the joint action of radiation and the high temperatures near the heated silica fiber. ${ }^{12}$ At the same time, the carbon layer which forms is also a strong radiation absorber across a wide range of wavelengths and this results in an interesting unstable mode of laser cutting. Preliminary experiments to study this effect on ex-vivo samples have demonstrated that the absorbing layer permits an increase in the effective resection speed of the laser scalpel by $0.97 \mu \mathrm{m} .{ }^{13}$ However, no comparative study has yet been undertaken of the scar characteristics during tissue regeneration after conventional laser resection and after resection using the absorbing coating, so the mechanism of the effect is not clear at this stage. 


\section{Materials and Methods}

We used two laser scalpels: an analogue of the Alta ${ }^{\circledR}$-ST DentalPhotonics (USA) ${ }^{14}$ operating in of $\mathrm{cw}$ generation mode, and a Nd:YAG pulse-periodic laser using a wavelength of $1.06 \mu \mathrm{m}$ and operating in spiking mode. The radiation was delivered to the tissue using replaceable silica fibers $320 \mu \mathrm{m}$ in diameter. We studied a carbon coating and an SAC based on silicone varnish. ${ }^{13}$ Two modes of contact tissue-cutting were investigated: one with a clear tip to the operating fiber and the other with the SAC tip. The contact exposure of animal integument to laser radiation with the fiber laser tips was performed by an experienced laser surgeon. The object under study was the skin of nonlinear white male rats in vivo.

The process of scar formation at the resection site was investigated only for the Alta ${ }^{\circledR}$-ST laser scalpel. Radiation with a power of $7 \mathrm{~W}$ was taken as being optimal as a result of data from Ref. 13 for the uncoated fiber. The same power was used for comparative analysis with the SAC fiber. The study was performed on 10 nonlinear white rats. Prior to resection, the animals were anaesthetized with $50 \mathrm{mg} / \mathrm{kg}$ of Zoletil ${ }^{\circledR}$ and then depilated in the lumbar region. The skin was dissected parallel to the spinal column using the clear fiber tip on one side, and the SAC tip on the other side. Morphological changes in the tissues surrounding the incision were assessed immediately after dissection and following 1, 5, 12 and 28 days. ${ }^{15}$ Five-micrometer sections were stained with hematoxylin and eosin. Two incisions were made at each time point for the scars resulting from both the clear fiber tip and from the SAC tip.

All in vivo experiments were carried out in accordance with the guidelines approved by the Ethical Committee of the Nizhny Novgorod State Medical Academy.

\section{Results}

\subsection{Exposure of skin to $N d: Y A G$ laser radiation}

Study of the exposure of the skin of experimental animals to Nd:YAG laser radiation in pulse-periodic mode (wavelength $1.06 \mu \mathrm{m}$, average radiation power $7 \mathrm{~W}$, pulse duration $200 \mu \mathrm{s}$, and pulse repetition rate $25 \mathrm{~Hz}$ ) showed that no incision was made as a result of contact exposure for $15-30 \mathrm{~s}$ with the clear fiber tip. Exposure to an SAC tip provided effective skin cutting with complete vessel coagulation. Histological investigation revealed (Fig. 2(a)) a tissue carbonization area $8-12 \mu \mathrm{m}$ thick, and (Fig. 2(b)) a coagulation area 500$600 \mu \mathrm{m}$ deep.

\subsection{Exposure of skin to Alta ${ }^{\circledR}-S T$ laser radiation}

Complete vessel coagulation occurred at skin cutting, but the resection speed with the clear fiber was $30 \%$ lower and the incision depth was nonuniform (see Fig. 1).

SAC initiated tip. Histological investigation showed an 800-940 $\mu \mathrm{m}$ deep incision with a layer of carbonized tissue 5-9 $\mu \mathrm{m}$ thick which was clearly visible one hour after dissection (Fig. 3). A heatinduced coagulation area containing unviable cells was formed around the dissection. The size of this area was about $1400-1600 \mu \mathrm{m}$. Twenty-four hours later, an area of secondary alterations with both viable and unviable tissue elements, had formed around the area of the coagulation changes. As a result, the width of the damaged tissue had increased to $2300-2800 \mu \mathrm{m}$. Leucocytic infiltration and edema were observed at the border between the damaged and normal skin. Five days after the exposure, a scab had formed and epitalization had been initiated. A section of rarefied connective tissue with randomly located, slightly swollen collagen
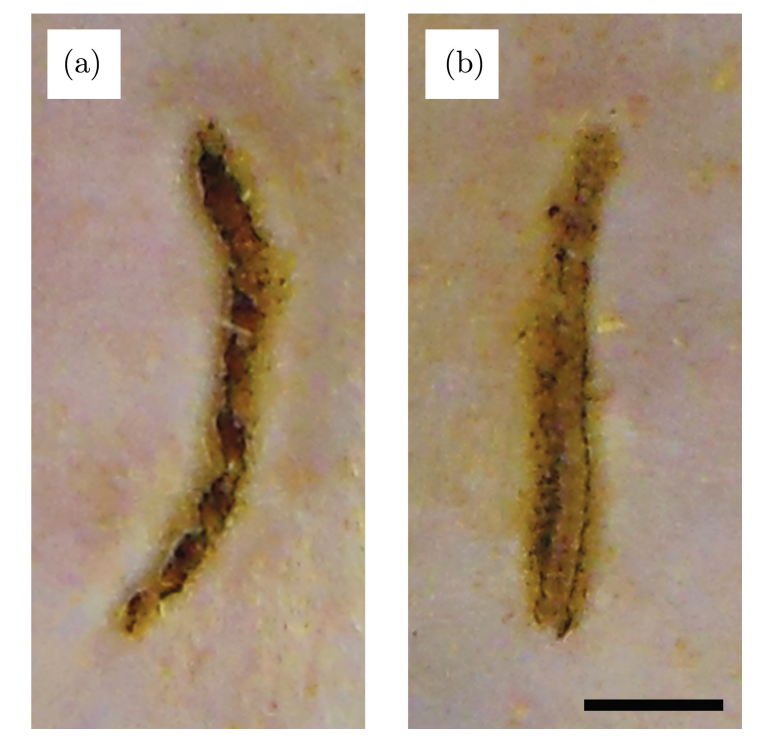

Fig. 1. Photo of the incision (a) without coating, (b) with SAC. 
fibers was observed under the newly formed epidermis. On the 12th day of the study, complete epitalization and the formation of a scar $1600 \mu \mathrm{m}$ wide and $230-330 \mu \mathrm{m}$ deep could be observed. Hair follicles of normal structure and a small number of vessels could be visualized in the scar tissue and around it 28 days after dissection, while the scar width had reduced to $650 \mu \mathrm{m}$.

Clear tip. In the samples taken one hour after dissection, the incision depth varied from $2400 \mu \mathrm{m}$ to $3000 \mu \mathrm{m}$, reaching the muscle fiber layer and even into it. The carbonization area was $16-38 \mu \mathrm{m}$, i.e., it was wider than the incision made by the SAP initiated tip. The coagulation area was 2100$2400 \mu \mathrm{m}$ across and $2400 \mu \mathrm{m}$ deep. Twenty-four hours after dissection the coagulation area had broadened and an area of secondary changes had appeared, having a total width of up to $3000 \mu \mathrm{m}$. The surrounding derma featured edema and leucocytic infiltration. On the fifth day a scab had appeared with a section of dermal destruction to a depth of $2400 \mu \mathrm{m}$. Twelve days after dissection, signs of wound epitalization had appeared along the edges, the defect width being 1300-1650 $\mu \mathrm{m}$. The depth of visible damage had also decreased to about $2000 \mu \mathrm{m}$. The formation of scar tissue could be observed in the deep layers. Twenty eight days after dissection, the scar had completely formed, had a width of 1400-1700 $\mu \mathrm{m}$ and occupied a region which reached as far as the muscle fibers. The scar tissue contained deformed hair follicles and only sporadic vessels.

\section{Discussion}

Our results for the determination of resection depth are comparable with the data obtained by other researchers. For instance, Karl Stock and coauthors reported similar results for diode laser radiation at a wavelength of $940 \mathrm{~nm}$ with the use of a cut bare fiber: $5 \mathrm{~W} / \mathrm{cw}: 711 \pm 268 \mathrm{~mm} ; 7 \mathrm{~W} / \bmod$.: $580 \pm 294 \mathrm{~mm}^{5}$

An important time aspect of the process under consideration is the time during which the laser radiation directly heats any given tissue site. This time is defined as $t=d / v$, where $d$ is the fiber diameter and $v$ is the cutting speed. In our case, $t \sim 1 \mathrm{~s}$. The thermal diffusivity length during time $t$, $l_{T}=\sqrt{\chi d / v}$ (where $\chi \approx 1.4 \times 10^{-3} \mathrm{~cm}^{2} / \mathrm{s}$ is the thermal diffusivity of the medium ${ }^{16}$ ), equals 300 $400 \mu \mathrm{m}$, which is more than the penetration depth of the incident radiation $l_{\lambda} \sim 1 / \alpha_{\text {scat }} \sim 100 \mu \mathrm{m}$. Hence, the localization region of the energy released as a result of the exposure to laser radiation is determined by the thermal diffusivity length. When a coated fiber is used, $20 \%$ of the radiation energy is released immediately on the tissue surface.
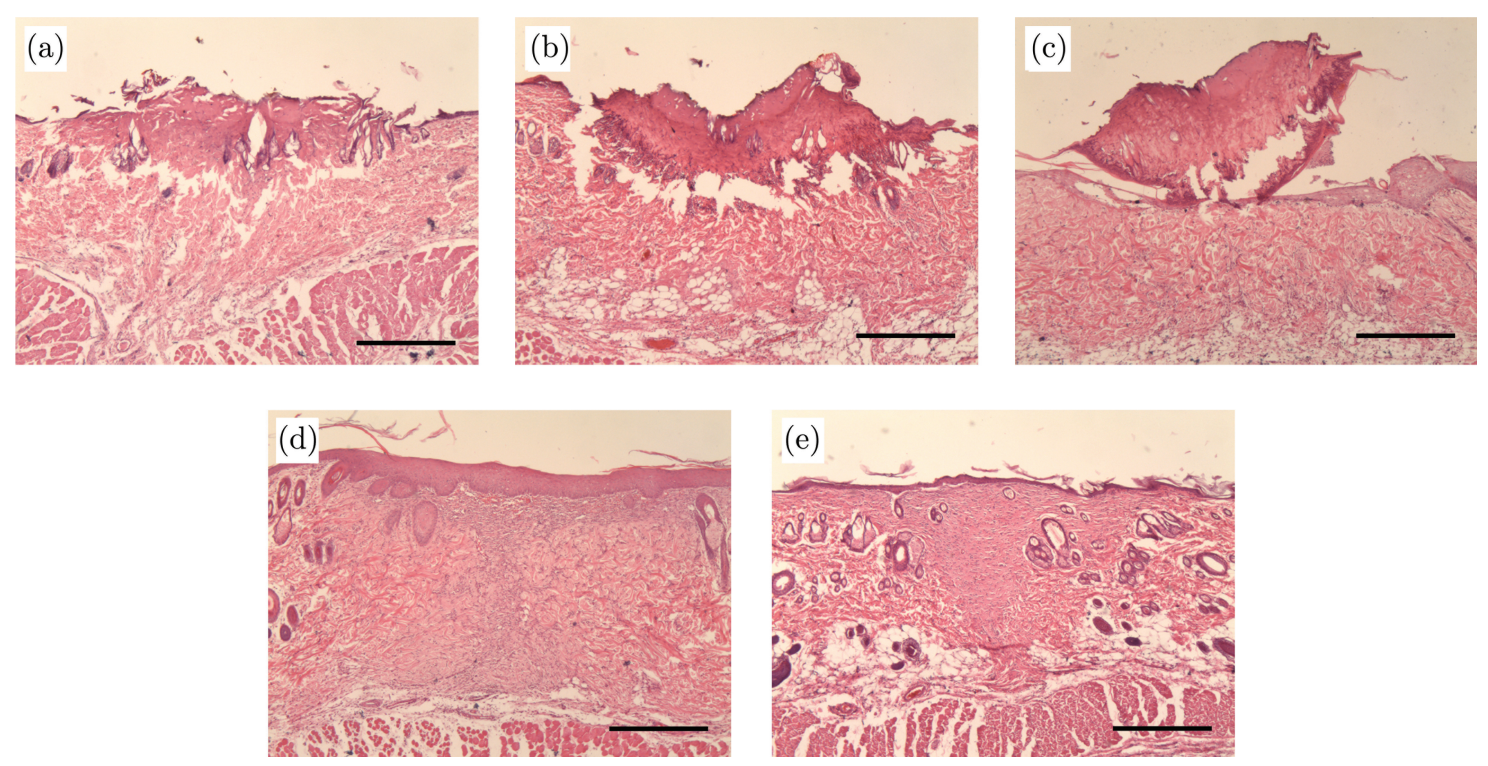

Fig. 2. Dynamics of tissue regeneration in the case of an SAC tip (a) immediately after exposure, (b) one day after, (c) five days after, (d) 12 days after, (e) 28 days after. Scale bar $500 \mu \mathrm{m}$. 

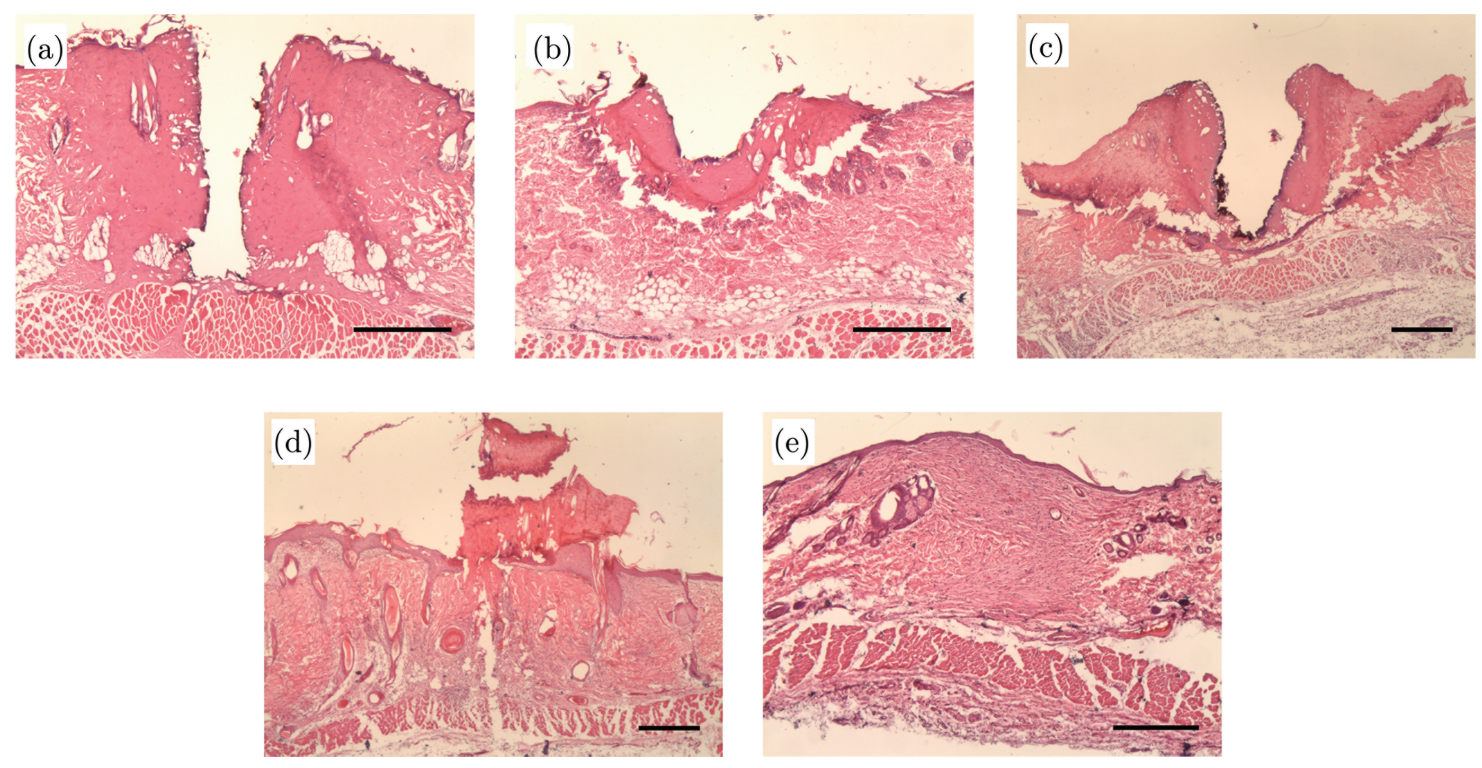

Fig. 3. Dynamics of tissue regeneration (a) immediately after exposure, (b) one day after, (c) five days after, (d) 12 days after, (e) 28 days after. Scale bar $500 \mu \mathrm{m}$.

Furthermore, a considerable part of the radiation energy escaping from the medium due to scattering is also released on the surface (see Fig. 4). Consequently, in this case, as compared to clear fiber tips, the laser energy is used more efficiently. For any given laser radiation power density, this allows a decrease in the time, $t$, needed to heat the medium surface to the required temperature. Such an increased rate of temperature rise increases the cutting speed, thereby resulting in a reduction of the thermal conductivity length and, hence, in a decreased area of coagulation. This was confirmed by the experimental data.

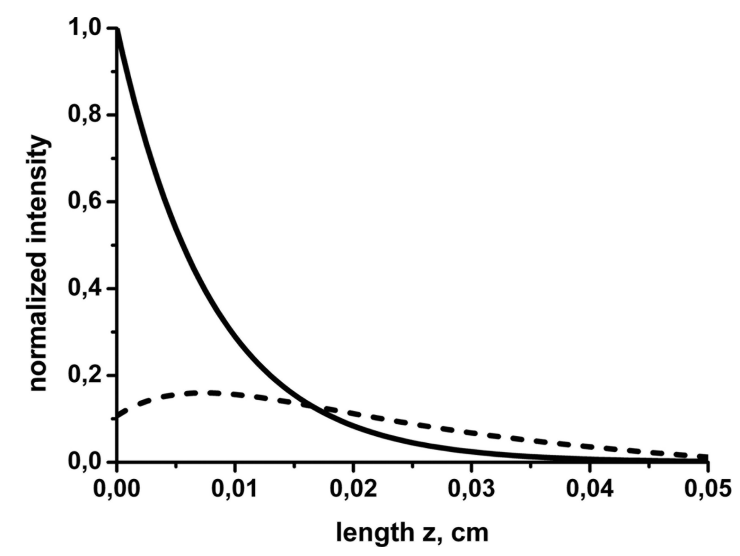

(a)
To get a better insight into the impact of absorption, consider the problem of light propagation in a strongly scattering medium. Let us take as an example a $500 \mu \mathrm{m}$ thick layer with a characteristic scattering coefficient of $120 \mathrm{~cm}^{-1}$ and an average scattering cosine of 0.9 , which corresponds to the known average values for human skin. ${ }^{16}$ As the exact value of the absorption coefficient is not known, we will consider a possible range of 0.14 $8 \mathrm{~cm}^{-1}$ for the values. ${ }^{17-19}$ Let us consider a case where the radiation being input through the front face of the sample in direct contact with a silica fiber $(n=1.49) 320 \mu \mathrm{m}$ in diameter, and suppose that

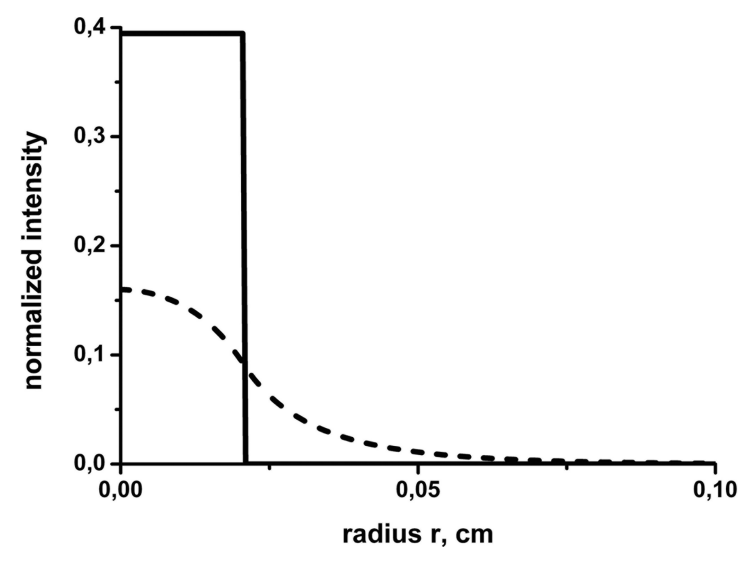

(b)

Fig. 4. Intensity distribution of incident (solid line) and scattered (dashed line) field components. (a) Distribution at the sample depth along the $r=0$ axis. (b) Radial distribution at a depth $z=75 \mu \mathrm{m}$ corresponding to the intensity maximum of the scattered field component. 


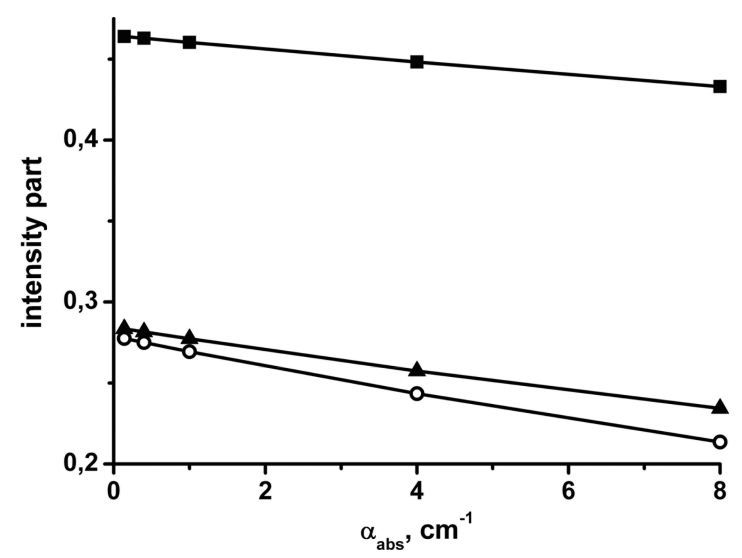

Fig. 5. Fraction of the scattered radiation returning to the fiber through the front face (line with squares), escaping through the front face outside the fiber (line with triangles), and escaping through the rear surface (line with circles) plotted against absorption coefficient.

the rear surface borders on a weakly scattering medium having an index of refraction the same as the medium under consideration $(n=1.4)$. The propagation of the scattering component is described in the following diffusion approximation.

The field intensity distribution along the sample depth at a point $r=0$ is presented in Fig. 4(a), and the radial distribution at the point $z=75 \mu \mathrm{m}$ is shown in Fig. 4(b) for the absorption coefficient $\alpha=8 \mathrm{~cm}^{-1}$. It is clear from the figure that, despite significant scattering, the radiation in the sample is strongly localized near the border, and a substantial part of the radiation is backscattered from the sample without being absorbed by it. Similar results have also been reported by other authors. ${ }^{20}$

The dependence of the fraction of the radiation escaping through different sample surfaces on the absorption coefficient is shown in Fig. 5. One can see in the figure that, with a pronounced change of the absorption coefficient, a considerable part of the scattered radiation returns to the region of the silica fiber.

Consequently, we can assume that as only part of the energy is absorbed in a strongly scattering medium, a substantial part of the radiation energy is not absorbed by the medium; instead, it is backscattered from it. Furthermore, a large proportion of the energy returns to the fiber. Thus, a fiber coated with an absorbing layer may provide more efficient heating due to the additional absorption of a considerable fraction of the radiation backscattered from the medium. Therefore, additional heating of the medium occurs due to its contact with the heated fiber.
Note, however, that the process of tissue surgery is accompanied both by some water evaporation and by carbonization, resulting in substantial changes in the properties of the tissues including optical ones. This makes modeling the process extremely complicated and demands special consideration.

In our case, tissue resection with a clear fiber tip was accompanied by instability caused by significant slowing of the cutting speed when the absorbing carbonized layer was disrupted during dissection. Dissection is more uniform when an SAC tip is used. Because of this instability, the parts of the resection trajectory are passed with a relatively low speed providing a significant increase in the dimensions of the thermally damaged zone, as follows both from the above discussion and as demonstrated by the experimental data.

\section{Conclusion}

Our research demonstrated that the application of an absorbing coating onto the silica fiber tip of a resection laser significantly changes the parameters of biotissue resection using it.

Contact exposure of white rat skin to radiation from a YAG:Nd laser at a wavelength of $1.06 \mu \mathrm{m}$ with an average radiation power of $7 \mathrm{~W}$ produced no incision when a clear fiber tip was used. Exposure to an SAC fiber tip using the same radiation parameters provided effective skin cutting.

Morphological monitoring after exposure to laser radiation with a wavelength of $0.98 \mu \mathrm{m}$ revealed that when resection was performed using the SAC fiber tip, the region of tissue damage was smaller than in the case of a clear tip. This, in turn, enabled tissue regeneration without the formation of a hard scar.

\section{Acknowledgment}

The work was supported by the Russian Science Foundation "The Use of Laser Induced Nonequilibrium Processes in Medical Technologies" No.1415-00840 dated 20 May 2014.

\section{References}

1. M. Freebody, "Fiber lasers at the cutting edge of surgery," Biophotonics (2013), Available at http:// www.photonics.com/Article.aspx?AID $=53575$. 
2. F. Beer, W. Körpert, H. Passow, A. Steidler, A. Meinl, A. G. Buchmair, A. Moritz, "Reduction of collateral thermal impact of diode laser irradiation on soft tissue due to modified application parameters", Lasers Med. Sci. 27, 917-921 (2012).

3. M. Judy, J. Matthews, B. Aronoff, D. Hults, "Soft tissue studies with $805 \mathrm{~nm}$ Diode Laser Radiation: Thermal effects with contact tips and comparison with effects of $1064 \mathrm{~nm} \mathrm{Nd}$ :YAG laser radiation", Laser Surg. Med. 13, 528-536 (1993).

4. P. S. Zubeev, A. Rotkov, I. Vaganov, I. Skvortsova, V. A. Kamensky, G. V. Gelikonov, A. M. Sergeev, "Resection of parenchymatous organs using pulse YAG: Nd laser radiation at $1.44-\mu \mathrm{m}$ and $1.32-\mu \mathrm{m}$ wavelengths", Proc. SPIE Lasers Surg. 3590, 439-443 (1999).

5. K. Stock, T. Stegmayer, R. Graser, W. Förster, R. Hibst, "Comparison of different focusing fiber tips for improved oral diode laser surgery", Lasers Surg. Med. 44, 815-823 (2012).

6. V. N. Kurlov, Sapphire: Propreties, growth and applications, Encyclopedia of Materials: Science and Technology, pp. 8259-8265, Pergamon, Elsevier Science Ltd (2001).

7. V. Saadatmanesh, H. M. G. Hussein, M. P. Loeb, S. Sulek, J. A. Milburn, "Method and apparatus for treating a body site with laterally directed laser radiation", Patent US 5242438 (1991).

8. V. P. Zharov, "New technology in surgery: Combination of laser and ultrasound", Proc. SPIE. 1892, 38-44 (1993).

9. G. E. Romanos, "Diode Laser Soft-Tissue Surgery: Advancement Aimed at Consistent Cutting, Improved Clinical Outcomes", Compend. Contin. Educ. Dent. 34, 752-757 (2013).

10. A. V. Shakhov, A. B. Terentjeva, V. A. Kamensky, L. B. Snopova, F. I. Feldstain, A. M. Sergeev, "Optical coherence tomography monitoring for laser surgery of laryngeal carcinoma", J. Surg. Oncol. 77, 253-259 (2001).

11. M. Amzayyb, R. R. van den Bos, V. M. Kodach, D. M. de Bruin, T. Nijsten, H. A. Neumann, M. J. C. van
Gemert, "Carbonized blood deposited on fibres during 810, 940 and 1,470 $\mathrm{nm}$ endovenous laser ablation: Thickness and absorption by optical coherence tomography", Lasers Med Sci. 25, 439-447 (2010).

12. V. I. Yusupov, V. M. Chudnovskii, V. N. Bagratashvili, Laser-Induced Hydrodynamics in Water and Biotissues Nearby Optical Fiber Tip, Hydrodynamics - Advanced Topics, Prof. Harry Schulz (Ed.), InTech, DOI: 10.5772/28517 (2011).

13. D. Kuznetsova, M. Karabut, V. Elagin, M. Shakhova, V. Bredikhin, O. Baskina, L. Snopova, A. Shakhov, V. Kamensky, "Comparative analysis of biotissue laser resection using strongly absorbing optical fiber tips", Opt. Photon. J. 5, 1-5 (2015).

14. http://altamls.com/applications/soft-tissue-surgery/how-top-surgery-works/.

15. S. Baranoski, E. A. Ayello, Wound Care Essentials: Practice Principles, Lippincott Williams \& Wilkins, Philadelphia, PA London, p. 479 (2008).

16. I. L. Shlivko, M. Y. Kirillin, E. V. Donchenko, D. O. Ellinsky, O. E. Garanina, V. A. Kamensky, "Identification of layers in optical coherence tomography of skin: Comparative analysis of experimental and Monte Carlo simulated images", Skin Res. Technol. 21, 419-425 (2015).

17. A. N. Bashkatov, E. A. Genina, V. I. Kochubey, V. V. Tuchin, "Optical properties of human skin, subcutaneous and mucous tissues in the wavelength range from 400 to $2000 \mathrm{nm",} \mathrm{J.} \mathrm{Phys.} \mathrm{D,} \mathrm{Appl.} \mathrm{Phys.}$ 38, 2543 (2005).

18. P. Taroni, A. Pifferi, A. Torricelli, L. Spinelli, G. M. Danesini, R. Cubeddu, "Do shorter wavelengths improve contrast in optical mammography?" Phys. Med. Biol. 49, 1203-1215 (2004).

19. A. V. Bykov, M. Y. Kirillin, A. V. Priezzhev, "Monte Carlo simulation of signals from model biological tissues measured by an optical coherence tomograph and an optical coherence doppler tomograph", Opt. Spectrosc. 101, 33-39 (2006).

20. H. P. Berlien, G. Müller, A. Lasermedizin, "Lehrund Handbuch für Praxis und Klinik", Ecomed, Landsberg München, Zürich (1989). 